\section{Nondiabetic renal disease is common in patients with type 2 diabetes}

A retrospective analysis from the US has reported a high prevalence of nondiabetic renal disease (NDRD) among diabetic patients undergoing renal biopsy.

Although patients with type 2 diabetes mellitus often experience diabetic nephropathy, they can also develop other renal diseases pathologically unrelated to diabetes. Nephrologists are, however, often reluctant to perform a renal biopsy-which would confirm the etiology of renal disease-in diabetic patients showing signs of nephropathy.

To determine the prevalence of NDRD in patients with type 2 diabetes, with or without evidence of diabetic glomerulosclerosis, Pham and co-workers reviewed the biopsy reports of 233 adults (mean age 58.1 \pm 13.7 years) who underwent renal biopsy at a single center during the period 1995-2005.

The researchers found that $53.2 \%, 27.5 \%$, and $19.3 \%$ of the patients had been diagnosed with NDRD, pure diabetic glomerulosclerosis, or concurrent NDRD and diabetic glomerulosclerosis, respectively. In patients with NDRD, the most common lesion was focal segmental glomerulosclerosis, seen in $21.0 \%$ of NDRD cases, followed by minimal-change disease (in $15.3 \%)$. In patients with concurrent NDRD and diabetic glomerulosclerosis, the most common lesions were IgA nephropathy, membranous glomerulonephritis and arterial/arteriolar nephrosclerosis, seen in $15.6 \%, 13.3 \%$ and $13.3 \%$ of cases, respectively. Absence of diabetic retinopathy was predictive of NDRD.

In light of the high prevalence of NDRD found in this study, the authors recommend that nephrologists maintain a low threshold of clinical suspicion for performing renal biopsy in diabetic patients. Early diagnosis of NDRD is crucial, as appropriate therapy could prolong renal survival in this patient population.

Original article Pham TT et al. (2007) Prevalence of nondiabetic renal disease in diabetic patients. Am J Nephrol 27: 322-328

\section{Eprodisate slows renal function decline in AA amyloidosis}

Deposition of fibrils in the kidneys of patients with amyloid $A(A A)$ amyloidosis can lead to proteinuria and progressive loss of renal function. Eprodisate inhibits fibril deposition in the kidneys of mouse models of AA amyloidosis, and Dember et al. recently reported that this agent might also delay progression of AA amyloidosis-related renal dysfunction in humans.

Their multicenter double-blind trial randomized 183 patients with AA amyloidosis and kidney involvement to either eprodisate $(n=89)$ or placebo $(n=94)$ twice daily for 24 months. Disease progression was defined as a $100 \%$ increase in serum creatinine level from baseline, a decrease in creatinine clearance of at least $50 \%$, or patient progression to end-stage renal disease or death.

In total, 124 patients (63 eprodisate and 61 placebo) completed 24 months of treatment. At the end of this period, disease was less likely to have worsened in eprodisate-treated patients than in those who received placebo ( $27 \%$ vs $40 \% ; P=0.06)$. Eprodisate treatment decreased the risk of worsening renal disease or death by about $40 \%(P=0.02)$, independent of baseline renal function and changes in serum AA level. This decreased risk was a result of eprodisate's effect on the progression of kidney dysfunction (but there was no significant effect on progression to end-stage renal disease); risks of death were similar in the two groups. Creatinine clearance rate decreased significantly faster in patients in the placebo group than in eprodisatetreated patients $(P=0.02)$. Adverse event profiles were similar in placebo and eprodisate groups.

Original article Dember LM et al. (2007) Eprodisate for the treatment of renal disease in AA amyloidosis. $\mathrm{N} \mathrm{Engl} \mathrm{J} \mathrm{Med}$ 356: $2349-2360$

\section{Whole-gene sequencing for diagnosis of type 1 primary hyperoxaluria}

Type 1 primary hyperoxaluria $(\mathrm{PH} 1)$, a rare inherited metabolic disorder, causes kidney stones and kidney damage or failure. The condition is caused by mutations in AGXT, the gene encoding the liver enzyme alanine-glyoxylate aminotransferase. There were 55 such mutations recorded in the Human Gene Mutation Database in 2004.

Monico et al. have sequenced the entire AGXT coding region in 55 unrelated probands with $\mathrm{PH} 1$. Most harbored two disease alleles. Gly170Arg was the most commonly detected mutation (37\% of alleles), followed 